\title{
Emotional and external eating and eating architecture in the UK
}

\author{
E. Magklis ${ }^{1}$, L.D. Howe ${ }^{2}$, T.M. Frayling ${ }^{3}$ and L. Johnson ${ }^{1}$ \\ ${ }^{1}$ Centre for Exercise, Nutrition and Health Sciences, University of Bristol, Bristol, England, UK, \\ ${ }^{2}$ MRC Integrative Epidemiology Unit, School of Social and Community Medicine, University of Bristol, Bristol, \\ England, UK and \\ ${ }^{3}$ Institute of Biomedical and Clinical Sciences, Peninsula Medical School, University of Exeter, Exeter, England, UK
}

Obesity is one of the most important public health challenges. Understanding the association between emotional and external eating (psychological traits that have been associated with adult weight gain) and eating architecture could highlight potential targets for intervention.

We evaluated cross-sectional associations between emotional and external eating with the size, frequency, variability of eating occasions and duration of eating among 1213 British adults participating in the UK National Diet and Nutrition Survey 2000-2001 with complete 7-day weighed dietary record data. Emotional and external eating (range 1-5) were assessed using the Dutch Eating Behaviour Questionnaire. Eating occasions were defined as all foods/drinks consumed at a unique time and were also classified as meals, snacks or drinks. Average frequency (occasions/day), size (kcal/occasion), eating period (minutes between first and last eating time) and variability (standard deviation of eating occasion size and daily eating frequency were calculated for meals, snacks, drinks and were regressed separately on external and emotional eating scores, adjusting for gender, age, BMI, restrained, emotional and external eating scores, social class, misreporting category and sleep duration.

Means (SD) for emotional and external eating scores were 1.80 (0.69) and $2.58(0.62)$ respectively. Adjusted regression models showed that a one-point higher emotional eating score was associated with $0.27(95 \% \mathrm{CI} 0 \cdot 12-0.42)$ more snack occasions/day, $0.38(95 \%$ CI 0.09-0.67) more eating occasions/day and a 10.25 kcal lower between-occasion SD (95\% CI - 17.34-3.16). A one-point higher external eating score was associated with larger snacks of, on average, $12.92 \mathrm{kcal}$ more (95\% CI 0.46-25.38), larger eating occasions of, on average, $9 \mathrm{kcal}$ more $(95 \%$ CI $0.82-17.22)$ and a $9.03 \mathrm{kcal}$ higher between-occasion SD (95\% CI 1.61-16.45), with a $4.41 \mathrm{kcal}$ higher between-drinks SD $(95 \%$ CI $0.39-8.43)$. A one-point higher emotional eating score was also associated with a longer eating period by, on average, 12.84 minutes (95\% CI 0.89-24.8). Associations were independent of BMI and other potential confounders.

Emotional eating was associated with more frequent intake within a day, mainly because of a larger number of snacks, eaten over a longer eating period, with their occasions varying less in caloric content. In contrast, external eating was associated with larger eating occasions, mainly because of the higher caloric content of snacks, and their eating occasions varying more in caloric content. The distinct patterns of eating architecture associated with external and emotional eating suggest different intervention targets should be explored further for their potential to prevent weight gain in people with differing eating behaviour traits.

E. Magklis is supported by a PhD studentship from the GW4 MRC Biomed DTP.

Koenders PG, van Strien T. Emotional eating, rather than lifestyle behaviour, drives weight gain in a prospective study in 1562 employees. Journal of Occupational \& Environmental Medicine. 2011;53(11), pp.1287-1293

Olea Lopez AL, Johnson L. Associations between Restrained Eating and the Size and Frequency of Overall Intake, Meal, Snack and Drink Occasions in the UK Adult National Diet and Nutrition Survey. PLoSOne. 2016;11(5):e0156320 